\title{
BioALBERT: A Simple and Effective Pre-trained Language Model for Biomedical Named Entity Recognition
}

Usman Naseem ( $\square$ usman.naseem@sydney.edu.au )

The University of Sydney https://orcid.org/0000-0003-0191-7171

Matloob Khushi

The University of Sydney

Vinay Reddy

The University of Sydney

Sakthivel Rajendran

The University of Sydney

Imran Razzak

Deakin University

Jinman Kim

The University of Sydney

Research article

Keywords: Named entity recognition, Biomedical text mining, Contextual word representations, Pre-trained Language Modes

Posted Date: October 13th, 2020

DOl: https://doi.org/10.21203/rs.3.rs-90025/v1

License: (c) (1) This work is licensed under a Creative Commons Attribution 4.0 International License.

Read Full License 


\title{
BioALBERT: A Simple and Effective Pre-trained Language Model for Biomedical Named Entity Recognition
}

Usman Naseem ${ }^{1}{ }^{*}$, Matloob Khushi $^{1}$, Vinay Reddy ${ }^{1}$, Sakthivel Rajendran ${ }^{1}$, Imran Razzak ${ }^{2}$ and Jinman Kim ${ }^{1,3}$

\begin{abstract}
Background: In recent years, with the growing amount of biomedical documents, coupled with advancement in natural language processing algorithms, the research on biomedical named entity recognition (BioNER) has increased exponentially. However, BioNER research is challenging as NER in the biomedical domain are: (i) often restricted due to limited amount of training data, (ii) an entity can refer to multiple types and concepts depending on its context and, (iii) heavy reliance on acronyms that are sub-domain specific. Existing BioNER approaches often neglect these issues and directly adopt the state-of-the-art (SOTA) models trained in general corpora which often yields unsatisfactory results.
\end{abstract}

Results: We propose biomedical ALBERT (A Lite Bidirectional Encoder Representations from Transformers for Biomedical Text Mining) - bioALBERT - an effective domain-specific pre-trained language model trained on huge biomedical corpus designed to capture biomedical context-dependent NER. We adopted self-supervised loss function used in ALBERT that targets on modelling inter-sentence coherence to better learn context-dependent representations and incorporated parameter reduction strategies to minimise memory usage and enhance the training time in BioNER. In our experiments, BioALBERT outperformed comparative SOTA BioNER models on eight biomedical NER benchmark datasets with four different entity types. The performance is increased for; (i) disease type corpora by $7.47 \%$ (NCBI-disease) and $10.63 \%$ (BC5CDR-disease); (ii) drug-chem type corpora by 4.61\% (BC5CDR-Chem) and 3.89\% (BC4CHEMD); (iii) gene-protein type corpora by $12.25 \%$ (BC2GM) and $6.42 \%$ (JNLPBA); and (iv) species type corpora by $6.19 \%$ (LINNAEUS) and $23.71 \%$ (Species-800) is observed which leads to a state-of-the-art results.

Conclusions: The performance of proposed model on four different biomedical entity types shows that our model is robust and generalizable in recognizing biomedical entities in text. We trained four different variants of BioALBERT models which are available for the research community to be used in future research.

Keywords: Named entity recognition; Biomedical text mining; Contextual word representations; Pre-trained Language Modes

\section{Background}

The growing volume of the published biomedical literature, such as clinical reports [1] and health literacy [2], are fuelling the advancements in the development of text mining algorithms. Biomedical named entity recognition (BioNER) intends to automatically identify biomedical entities such as diseases, chemicals, genes and proteins, etc., from the biomedical literature. So, a crucial step towards this aim is to build bet-

${ }^{*}$ Correspondence: usman.naseem@sydney.edu.au

${ }^{1}$ School of Computer Science, University of Sydney, Australia

Full list of author information is available at the end of the article ter and effective methods which can automatically recognize and extract biomedical entities. BioNER is an integral component of many downstream information extraction tasks e.g., disease-treatment [3] relations and extracting drug-to-drug interactions [4]. Traditionally, BioNER relies on feature engineering methods (e.g., lexicon-based, rules-based and statistics-based). However, feature engineering is dependent on domainspecific knowledge which does not perform well on BioNER [5].

Deep learning (DL) with its ability to automatically extract features have become common in BioNER 
recently [6]. For instance, Long Short-Term Memory (LSTM) is usually employed to learn vector representations of each word in a sentence, and then as the input to conditional random fields (CRF) have considerably increased the performance in BioNER [6]. Recently state-of-the-art (SOTA) DL based language models for instance Embeddings from Language Models (ELMo) [7], Bidirectional Encoder Representations from Transformers (BERT) [8] and (A Lite Bidirectional Encoder Representations from Transformers (ALBERT) [9] obtained SOTA best performance on many NLP tasks.

Although these models show promising results, but applying them on BioNER has multiple challenges and limitations: (i) a limited amount of training data; (ii) an entity could represent multiple entity types depending on its textual context, i.e., a "BRCA1" can be referred as gene name as well as a disease entity depending on its context. Similarly, "heart attack" and "myocardial infarction" refer to the same concept and, (iii) the heavy use of acronyms in biomedical texts makes it challenging to identify concepts, i.e., the abbreviation " RA" may refer to "right atrium," "rheumatoid arthritis," or one of several other concepts, where the resolution of the abbreviation is, therefore, contextdependent. Therefore, current models in BioNER rely on various context-independent and transformer-based context-dependent language models which are trained on biomedical corpora $[10,11,12]$.

To overcome the identified limitations, we present biomedical ALBERT - bioALBERT - a contextdependent, fast and effective language model that addresses the shortcomings of recently proposed domainspecific language models. BioALBERT is trained on large biomedical corpora which address the limitation of limited training data. We also innovate in the adoption of cross-layer parameter sharing by learning parameters for the first block and reuse the block in the remaining layers instead of learning unique parameters for each of the layers and sentence-order-prediction (SOP) technique as a measure of coherence loss between sentences. SOP takes two consecutive sentences from training data and creates a random pair from different sentences which helps the model to learn better representations and finally, in BERT based models the size of the embedding was linked to the hidden layer sizes of the transformer blocks. These embeddings are projected directly to the hidden space of the hidden layer whereas in our model we use factorized embedding parameterization which decomposes embedding matrix in two small matrices which distinguish the size of the hidden layers from the size of vocabulary embeddings. This allows for increasing the hidden size without significantly increasing the parameter size of the vocabulary embeddings. BioALBERT is simple and efficient when fine-tuned for BioNER task as compared to other SOTA models. We evaluate our model on eight biomedical named entity recognition benchmark datasets.

In biomedical text mining research, there is a long history of using shared language representations to capture the semantics of the text. One established trend is a form of word-embeddings [13] that represent syntactic and semantic meaning, and map words into low-dimensional vectors. Similar methods also have been derived for improving embeddings of word sequences by introducing sentence embeddings [14]. These context-independent word embeddings approach such as word2vec [13] were trained on biomedical corpus containing words along with phrases that are not normally available in a general domain corpus [10]. These methods always require complex neural networks to be effectively used and model context-independent representations.

Another common trend, particularly in recent years, is text representation based on context $[7,8,9]$. Unlike traditional word-embeddings, this enables a word to change its meaning depending on the context in which it occurs. Several other works have investigated the benefit of contextual models in biomedical and clinical areas. Several researchers trained ELMo on biomedical corpora and presented BioELMo and found that BioELMo beats ELMo on BioNER tasks $[11,15]$. Along with their work, a pre-trained BioELMo model was published, enabling further clinical research. Beltagy et al. [16] released Scientific BERT (SciBERT), where BERT was trained on the scientific texts. In non-contextual embedding, BERT has been usually superior and better than ELMo.

Si et al. [17], trained the BERT on clinical notes corpora, using complex task-specific models to improve both traditional embedding and ELMo embedding on the i2b2 2010 and 2012 BioNER. Similarly in another study, a new domain-specific language model, BioBERT [12], trained a BERT model on biomedical documents from PMC abstracts ${ }^{[1]}$ and articles from PubMed ${ }^{[2]}$ that resulted in improved BioNER results. Peng et al [18] introduced Biomedical Language Understanding Evaluation (BLUE), a collection of resources for evaluating and analysing biomedical natural language representation models. Their study also confirmed that pre-trained BERT models with PubMed abstracts and clinical notes perform better than many other state-of-the-art models.

Despite this success, BERT has some limitations such as BERT has a huge number of parameters which is the cause for problems like degraded pre-training

${ }^{[1]}$ https://www.ncbi.nlm.nih.gov/pmc/

${ }^{[2]}$ https://www.ncbi.nlm.nih.gov/pubmed/ 
time, memory management issues and model degradation etc [9]. These issues are very well addressed in ALBERT, which is modified based on the architecture of BERT and proposed by Lan et al. [9]. In scaling pretrained models, ALBERT implements two-parameter reduction methods that lift the essential barriers. (i) factorized embedding parameterization - decomposes the large vocabulary embedding matrix into two small matrices, (ii) replaces the NSP loss by SOP loss; and (iii) cross-layer parameter sharing- stops the parameter from prospering with the network depth. These methods significantly lower the number of parameters used when compared with BERT without significantly affecting the performance of the model, thus improving parameter-efficiency. An ALBERT configuration is same as BERT (large) has 18 times less parameters and can be trained about 1.7 times faster.

Fine-tuning has been successfully used to transfer pre-trained weights as initialisation for parameters for various downstream tasks [19]. This improves the efficiency of the target task when we have limited data and similar tasks [20]. Recently, fine-tuning of pretrained language models have been widely utilized in various text mining tasks [21]. Liu et al. [22] pretrained LSTM with a language model and fine-tuned it, and this has contributed to improved performance for various text classification tasks.

Universal Language Model Fine-tuning (ULMFiT) used general-domain pre-training and fine-tuning techniques to avoid over-fitting and achieved SOTA performance on the datasets with less samples [19]. Similarly, authors of BERT, ALBERT, BioBERT and others tested the performance of their model for a wide range of tasks with minimum fine-tuning efforts and achieved good performance. Recently, multi-task finetuning has led to improvements even with many target tasks [23]. In this paper, we fine-tuned our pretrained language model BioALBERT which is trained on biomedical corpora for BioNER. BioALBERT offers better performance on BioNER as it addresses the shortcomings of BERT used in BioBERT. However, this is not a trivial task as it requires several optimisations which are discussed in the next section. Our pre-trained models, along with the source code, will be publicly available.

\section{Results}

In this section, we present the dataset used, baselines and evaluation to demonstrate the effectiveness of our model.

\section{Datasets}

Our model is evaluated on eight biomedical NER benchmark datasets which contain four types of entities and provided by Lee et al. [12]. For (i) Disease
Table 1 Statistics of the BioNER datasets

\begin{tabular}{|c|c|c|}
\hline Entitity Type & Dataset & \# of Annotations \\
\hline \multirow{2}{*}{ Disease } & NCBI Disease & 6,881 \\
\cline { 2 - 3 } & BC5CDR & 12,694 \\
\hline \multirow{2}{*}{ Drug/Chem } & BC5CDR & 15,411 \\
\cline { 2 - 3 } & BC4CHEMD & 79,842 \\
\hline \hline \multirow{2}{*}{ Drug/Protein } & BC2GN & 20,703 \\
\cline { 2 - 3 } & JNLPBA & 35,460 \\
\hline \hline \multirow{2}{*}{ SPECIES } & LINNAEUS & 4,077 \\
\cline { 2 - 3 } & Species-800 & 3,708 \\
\hline
\end{tabular}

entity type, we used NCBI-Disease [30] and BC5CDRDisease [31] datasets; (ii) Drug-Chem entity type, we used BC5CDR-Chem [31] and BC4CHEMD [32] datasets; (iii) Drug-Protein entity type, we used BC2GM [33] and JNLPBA [34] datasets; and (iv) Species entity type, we used LINNAEUS [35] and Species-800 [36] datasets. The statistics of the BioNER datasets used in this study are given in Table 1.

\section{Baselines}

To assess the performance of the proposed method, an exhaustive comparison is performed with several advanced SOTA methodologies along with their published results ${ }^{[3]}$. Our model is compared with the following methods.

Yoon et al. [28] presented CollaboNet which contains several BiLSTM and CRF models for BioNER. Previously proposed models could only handle datasets with a single form of entity whereas CollaboNet leverages different datasets and demonstrated the good performance. CollaboNet is based on several single task models for named entity recognition that transmit information to one another for better predictions.

Lou et al. [26] introduced transition based algorithm for the normalization and disease entity recognition. Their model was built on the transition structured prediction framework. In another study, an attention based BiLSTM-CRF, was proposed by Lou et al. [27] for chemical named entity recognition (CNER). This approach leverages global document-level information gathered through the attention process to ensure continuity of labelling across several cases of the similar token in a document and achieved good results with some feature engineering.

$\mathrm{Xu}$ et al. [24] introduced DABLC, a new dictionarybased and document-level attention approach for disease entity recognition. At document level, DABLC labels the consistency of several instances in a text and incorporates an external dictionary of diseases which

${ }^{[3]}$ The reported results were obtained from the original publication. 
Table 2 Comparison of performance in biomedical named entity recognition (BioNER) task.

\begin{tabular}{|c|c|c|c|c|c|c|c|c|c|c|c|}
\hline \multirow{3}{*}{ Type } & \multirow{3}{*}{ Datasets } & \multirow{3}{*}{ M } & \multirow{3}{*}{ SOTA } & BioBERT v1.0 & BioBERT v1.0 & BioBERT v1.0 & BioBERTv1.1 & BioALBERT 1.0 & BioALBERT 1.0 & BioALBERT 1.1 & BioALBERT 1.1 \\
\hline & & & & (PubMed) & (PMC) & (PubMed+PMC) & (PubMed) & (PubMed) & (PubMed+PMC) & (PubMed) & (PubMed+PMC) \\
\hline & & & & Base & Base & Base & Base & Base & Base & Large & Large \\
\hline \multirow{6}{*}{ Disease } & \multirow{3}{*}{ NCBI Disease } & $\mathrm{p}$ & 88.30 & 86.76 & 86.16 & 89.04 & 88.22 & 97.45 & 96.84 & 97.18 & 97.38 \\
\hline & & $\mathrm{R}$ & 89.00 & 88.02 & 89.48 & 89.69 & 91.25 & 94.39 & 94.40 & 97.18 & 94.37 \\
\hline & & $\mathrm{F}$ & 88.60 & 87.38 & 87.79 & 89.36 & 89.71 & 95.89 & 95.61 & 97.18 & 95.85 \\
\hline & \multirow{3}{*}{ BC5CDR } & $\mathrm{p}$ & 89.61 & 85.80 & 84.67 & 85.86 & 86.47 & 99.69 & 99.11 & 99.27 & 99.39 \\
\hline & & $\mathrm{R}$ & 83.09 & 86.60 & 85.87 & 87.27 & 87.84 & 95.72 & 96.17 & 96.33 & 95.85 \\
\hline & & $\mathrm{F}$ & 86.23 & 86.2 & 85.27 & 86.56 & 87.15 & 97.66 & 97.62 & 97.78 & 97.61 \\
\hline \multirow{6}{*}{ Drug/Chem } & \multirow{3}{*}{ BC5CDR } & $\mathrm{p}$ & 94.26 & 92.52 & 92.46 & 93.27 & $\overline{93.68}$ & 99.99 & 99.99 & 99.99 & 99.99 \\
\hline & & $\mathrm{R}$ & 92.38 & 92.76 & 92.63 & 93.61 & 93.26 & 95.89 & 96.24 & 95.62 & 95.68 \\
\hline & & $\mathrm{F}$ & 93.31 & 92.64 & 92.54 & $\overline{93.44}$ & 93.47 & 97.9 & 98.08 & 97.76 & 97.79 \\
\hline & \multirow{3}{*}{ BC4CHEMD } & $\mathrm{p}$ & 92.29 & 91.77 & 91.65 & 92.23 & 92.80 & 97.76 & 97.71 & 97.71 & 97.88 \\
\hline & & $\mathrm{R}$ & 90.01 & 90.77 & 90.30 & 90.61 & 91.92 & 94.22 & 94.83 & 94.83 & 94.63 \\
\hline & & $\mathrm{F}$ & 91.14 & 91.26 & 90.97 & 91.41 & 92.36 & 95.96 & 96.25 & 96.25 & 96.23 \\
\hline \multirow{6}{*}{ Drug/Protein } & \multirow{3}{*}{$\mathrm{BC} 2 \mathrm{GM}$} & $\mathrm{p}$ & 81.81 & 81.72 & 82.86 & 85.16 & 84.32 & 97.86 & 97.84 & 98.26 & 98.02 \\
\hline & & $\mathrm{R}$ & 81.57 & 83.38 & 84.21 & $\overline{83.65}$ & 85.12 & 94.87 & 94.27 & 95.72 & 94.70 \\
\hline & & $\mathrm{F}$ & 81.69 & 82.54 & 83.53 & 84.40 & 84.72 & 96.34 & 96.02 & 96.97 & 96.33 \\
\hline & \multirow{3}{*}{ JNLPBA } & $\mathrm{p}$ & 74.43 & 71.11 & 71.17 & 72.68 & 72.24 & 85.14 & 85.60 & 86.23 & 85.56 \\
\hline & & $\mathrm{R}$ & 83.22 & 83.11 & 82.76 & 83.21 & 83.56 & 80.43 & 80.98 & 81.90 & 81.49 \\
\hline & & $\mathrm{F}$ & 78.58 & 76.65 & 76.53 & 77.59 & 77.49 & 82.72 & 83.22 & 84.01 & 83.53 \\
\hline \multirow{6}{*}{ Species } & \multirow{3}{*}{ LINNAEUS } & $\mathrm{p}$ & 92.80 & 91.83 & 91.62 & 93.84 & 90.77 & 99.95 & 99.98 & 99.98 & 99.92 \\
\hline & & $\mathrm{R}$ & 94.29 & 84.72 & 85.48 & 86.11 & 85.83 & 99.47 & 99.44 & 99.48 & 99.55 \\
\hline & & $\mathrm{F}$ & 93.54 & 88.13 & 88.45 & 89.81 & 88.24 & 99.71 & 99.72 & 99.73 & 99.73 \\
\hline & \multirow{3}{*}{ Species-800 } & $\mathrm{p}$ & $\overline{74.34}$ & 70.60 & 71.54 & 72.84 & 72.80 & 99.17 & 98.93 & 99.10 & 98.75 \\
\hline & & $R$ & 75.96 & 75.75 & 74.71 & 77.97 & 75.36 & 98.34 & 98.04 & 98.95 & 98.69 \\
\hline & & $\mathrm{F}$ & 74.98 & 73.08 & 73.09 & 75.31 & 74.06 & 98.76 & 98.49 & 99.02 & 98.72 \\
\hline
\end{tabular}

Notes: Metrics (M), Precision (P), Recall $(R)$ and $F 1(F)$ results are recorded on each dataset. Bold has the best results, and underlined has the second best results. We report the SOTA model results on various datasets as follows: NCBI-Disease results from Xu et al. [24], BC2GM from Sachan et al. [25], BC5CDR-disease from Lou et al. [26], BC4CHEMD from Luo et al. [27], BC5CDR-chemical and JNLPBA from Yoon et al. [28], and LINNAEUS and Species-800 from Giorgi and Bader [29].

is designed with five disease databases comprising a rich set of disease entities.

Lee et al. [12] proposed a biomedical BERT (BioBERT) which is pre-trained language model trained on biomedical corpus. Authors of BioBERT model demonstrated that training BERT on biomedical corpus improve the performance on BioNER and outperforms previously presented models for BioNER. We compare BioALBERT with both BioBERT (v1.0 and V1.1) models and other SOTA models used in BioNER task. We chose these methods because they are the SOTA, and exhibit the highest performance on the basis of the meta-analysis carried out.

\section{Evaluation}

Table 2 presents the performance of all the variants of BioALBERT and contrasts them to baseline methodologies. Our model outperforms all other methods on all eight datasets. For, (i) Disease-type datasets, BioALBERT increased the performance by by $7.47 \%$ (NCBI-disease) and 10.63\% (BC5CDR-disease); (ii) drug-chem type corpora by $4.61 \%$ (BC5CDR-Chem) and $3.89 \%$ (BC4CHEMD); (iii) gene-protein type corpora by $12.25 \%$ (BC2GM) and $6.42 \%$ (JNLPBA); and (iv) species type corpora by $6.19 \%$ (LINNAEUS) and $23.71 \%$ (Species-800) is observed which is the SOTA performance on BioNER.

We have performed multiple comparisons to analyse the effectiveness of BioALBERT as compared to BioBERT. We measured and compared the training time of BioALBERT with BioBERT. We found that all models BioALBERT outperforms BioBERT with a

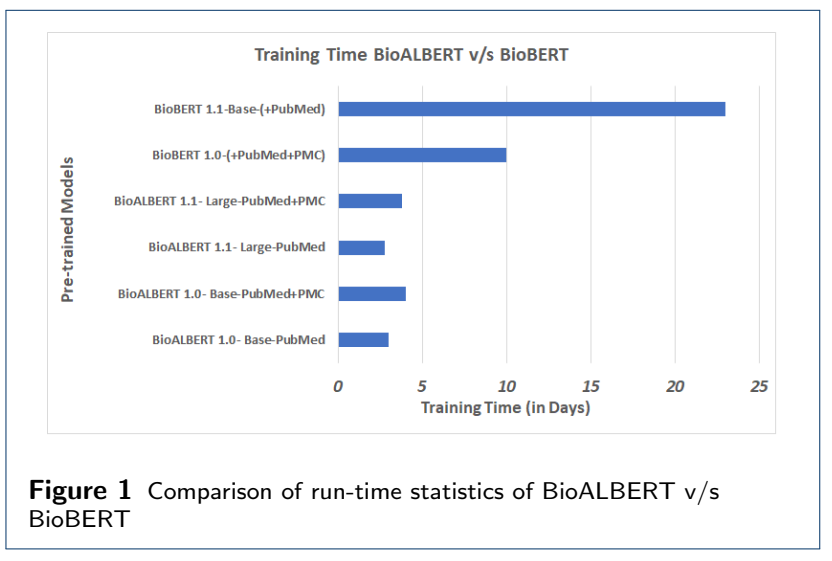

considerable margin which makes it more faster and practical as compared to BioBERT models. The run time statistics of both pre-trained models are given in Figure. 1. We also compared the performance comparison (shown in Figure 2 ) of the same versions (trained on same corpora and for the same number of steps) of both BioALBERT and BioBERT. We can see that in Figure (2a), we compared BioBERT v1.0 base model which is trained on PubMed with BioALBERT 1.0 base model trained on Pubmed and similarly in Figure (2b), we compared BioBERT v1.0 base model trained on Pubmed and PMC with BioALBERT 1.0 base model trained on PubMed and PMC biomedical corpus. In both cases, BioALBERT outperformed BioBERT on all of the eight datasets which makes our model more robust, generalize and effective than BioBERT. 


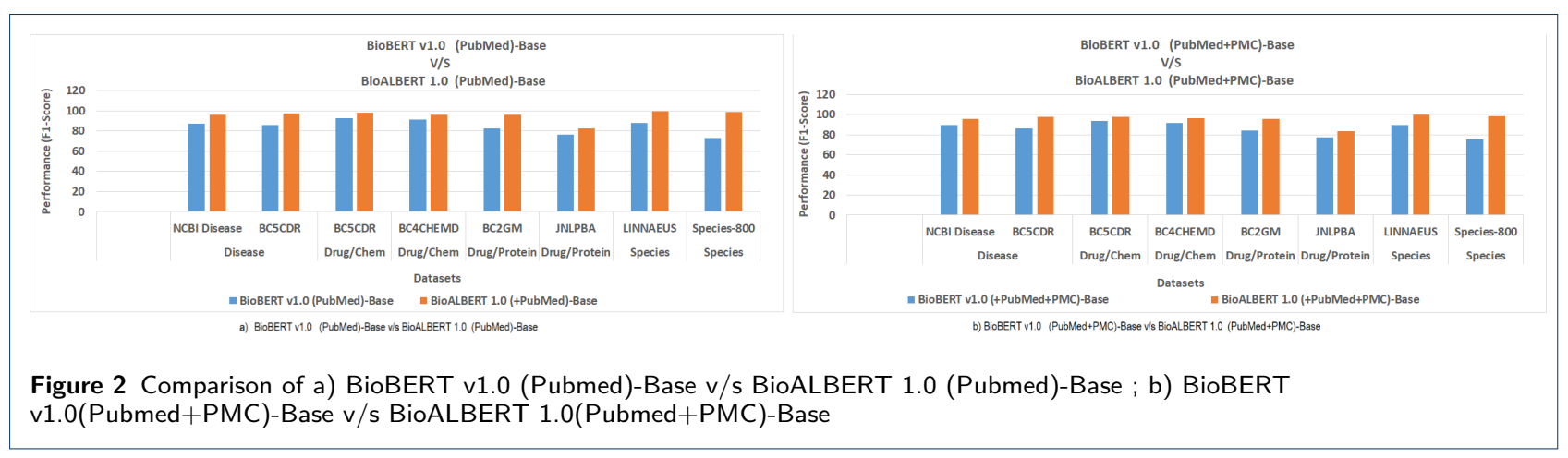

\section{Discussions}

BioALBERT gives better performance and addresses the previously mentioned challenges in the biomedical domain. We attribute this to the BioALBERT built on top of the transformer-based language model that learns contextual relationship between words in the corpus. The transformer encoder learns the whole sequence of tokens at once when compared to directional algorithms which learns the input tokens sequentially (i.e., right-to-left or left-to-right). This feature makes it possible for the algorithm to learn meaning of a word based on contexts (i.e., left and right of the word) and to address the contextual representation problem.

Our model addresses the shortcomings of BERT based biomedical models. At first, BioALBERT uses cross-layer parameter sharing and reduces 110 million parameters of 12-layer BERT-base model to 31 million parameters while keeping the same number of layers and hidden units by learning parameters for the first block and reuse the block in the remaining 11 layers. Secondly, our model uses the SOP, which takes two segments from the training corpus that appear consecutively and constructs a random pair of segments from different documents. This enables the model to learn about discourse-level coherence characteristics from a finer-grained distinction and leads to better learning representation in downstream tasks. Thirdly, our model uses factorized embedding parameterization to decompose the embedding matrix into two small matrices, the smaller size layer vocabulary and hidden layer minimise the parameters number between both the vocabulary and first hidden layer whereas in BERT based biomedical models embedding size is equal to the size of the hidden layer. Finally, our model is trained on massive biomedical corpus to be effective on BioNER to address the issue of the shift of word distribution from general domain corpus to biomedical corpus. All these, when combined, address all the issues associated with BioNER stated earlier. As our proposed model provides a consistent gain in performance for all tested datasets over all other models,

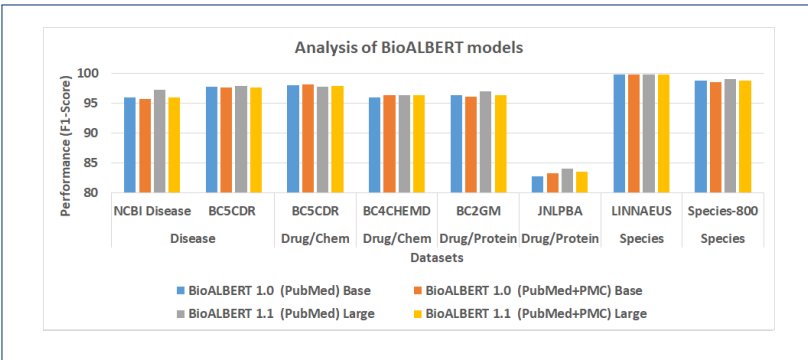

Figure 3 Comparison of different variants BioALBERT models

we can infer that our model is a robust solution for BioNER task.

To extend our analysis, we analysed the performance of different pre-trained models of BioALBERT. We found out that the performance of all BioALBERT models are almost equally good, but BioALBERT 1.1 Large trained on PubMed works better than others (shown in Figure. 3). BioALBERT 1.1 Large model, trained on PubMed with dup-factor as five gives better performances. This demonstrates that the relevance of duplication data in NLP tasks.

\section{Conclusion}

In this study, we presented BioALBERT, a pre-trained language model for biomedical named entity recognition. We presented four different variants of BioALBERT models which are trained on huge biomedical corpora for a different number of steps. We demonstrated that training ALBERT on biomedical corpus is an essential step in applying it to BioNER. As future works, we plan to pre-trained other versions which include hybrid of general and biomedical corpora of ALBERT on biomedical corpora with more training steps and fine-tune on biomedical text mining task. We also plan to fine-tune BioALBERT on other text mining tasks to show the effectiveness of our model.

\section{Methods}

First, we initialized BioALBERT with weights from ALBERT. BioALBERT is trained on biomedical do- 


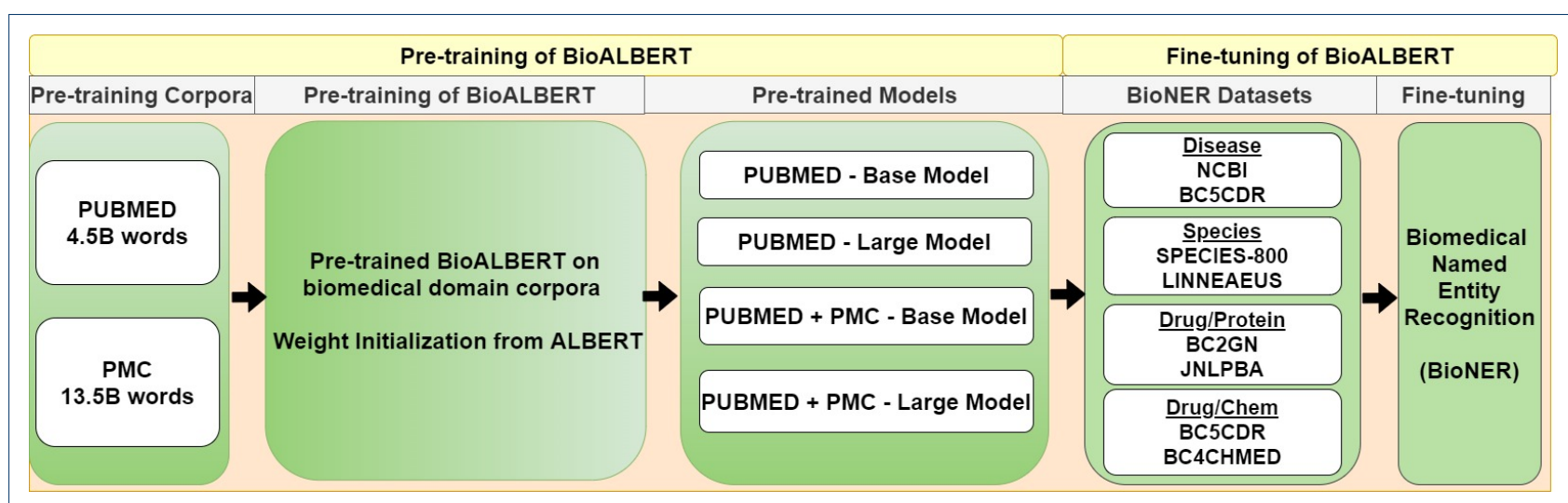

Figure 4 Overview of the pre-training and fine-tuning of BioALBERT on NER

main corpus (PubMed and PMC). To demonstrated the effectiveness of our model, BioALBERT is finetuned on BioNER. We tested various pre-training methods with various combinations and sizes of biomedical corpus. The overview of our methodology is shown in Figure. 4. Below we present details of each step involved in pre-training and fine-tuning of BioALBERT.

\section{Pre-training of BioALBERT}

In this section, we present steps involved in the pretraining of BioALBERT, which has the same architecture as ALBERT, which makes it simple. BioALBERT is trained on abstracts from PubMed and fulltext articles of PMC which contains biomedical terms and enables to train the ALBERT model, trained on the general text on biomedical corpora. Unstructured and raw biomedical corpora (PubMed and PMC) was converted to structured format by converting raw text files into a single sentence in which; (i) Within a text, all blank lines are removed and transformed into a single paragraph; (ii) any line with a length of less than 20 characters is excluded and (iii) there will be a blank line (when the different files are combined) between each document for training the model. Overall, PubMed contains approximately 4.5 Billion words, and PMC contains about 13.5 Billion words. As an initial step, we have initialized the model weights from ALBERT to create BioALBERT model by pre-training on biomedical corpora and kept the original vocabulary of ALBERT.

Sentence embeddings are used for tokenization, and for that, we pre-processed the data as a sentence text. So every line in the input text document is a sentence, and an empty line separates every document. We set the maximum length of each sentence to 512 words. Shorter sentences were padded to make 512 whereas longer sentences were truncated. Learning rate ( $\mathrm{lr}=$ 0.00176) and 3,125 warm-up steps are used for training all of our models except for BioALBERT v 1.1
Table 3 Summary of parameters used in the Pre-training

\begin{tabular}{|c|c|c|}
\hline \multicolumn{3}{|c|}{ Summary of All parameters used: (Pre-Training) } \\
\hline Name & BioALBERT 1.0 & BioALBERT 1.1 \\
\hline Architecture & ALBERT Base & ALBERT Large \\
\hline Activation Function & GeLU & GeLU \\
\hline Attention Heads & 12 & 16 \\
\hline No. of Layers & 12 & 24 \\
\hline Size of Hidden Layer & 768 & 1,024 \\
\hline Size of Embedding & 128 & 128 \\
\hline Size of Vocabulary & $30 \mathrm{k}$ & $30 \mathrm{k}$ \\
\hline Optimizer Used & LAMB & LAMB \\
\hline Training Batch Size & 1024 & 256 \\
\hline Evaluation Batch Size & 16 & 16 \\
\hline Maximum Sentence Length & 512 & 512 \\
\hline Maximum Predictions per Sentence & 20 & 20 \\
\hline Learning Rate (Ir) & 0.00176 & 0.00062 \\
\hline Training Steps for PubMed & $200 \mathrm{~K}$ & $200 \mathrm{~K}$ \\
\hline Training Steps for PMC & $270 \mathrm{~K}$ & $270 \mathrm{~K}$ \\
\hline Warm-up Steps & 3,125 & 3,125 \\
\hline
\end{tabular}

which trained on Pubmed and PMC where learning rate $(\mathrm{lr}=0.00062)$ is used to stop the issue of exploding loss. Both BioALBERT 1.0 and BioALBERT 1.1 are trained for $200 \mathrm{~K}$ and $270 \mathrm{~K}$ steps on PubMed and PMC corpus respectively. We used LAMB optimizer during training process of our models and kept the vocabulary size to $30 \mathrm{~K}$. GeLU activation is used in all variants of models during training process. For BioALBERT 1.1 base models training batch size of 1,024 was used where as in BioALBERT 1.1 large models training size was reduced to 256 due to computational resources limitations. Summary of parameters used in the training process is given in Table 3.

Table 4 BioALBERT Pre-trained Models

\begin{tabular}{|c|c|c|c|c|}
\hline Model Version & Model & Trained On & \# of words & Steps \\
\hline BioALBERT 1.0 & Base & PubMed & $4.3 \mathrm{~B}$ & $200 \mathrm{~K}$ \\
\hline BioALBERT 1.0 & Base & PubMed+PMC & $18.8 \mathrm{~B}$ & $470 \mathrm{~K}$ \\
\hline BioALBERT 1.1 & Large & PubMed & $4.3 \mathrm{~B}$ & $200 \mathrm{~K}$ \\
\hline BioALBERT 1.1 & Large & PubMed+PMC & $18.8 \mathrm{~B}$ & $470 \mathrm{~K}$ \\
\hline
\end{tabular}

We experimented with different settings and found out that both (base and large) pre-trained models were successful with bigger batch size on V3-8 TPU. We have used two different embedding sizes, i.e., 128 and 
256. The 128 embedding size creates a base model with 12 Million parameters, whereas a large model has 16 Million parameters with 256 embedding size. With these combinations, we have presented a total of four models, given in Table 4 .

\section{Fine-tuning of BioALBERT}

Fine-tuning of BioALBERT on BioNER task is presented in this section. BioNER involves annotating tokens in a sentence as named-entities. The labelled datasets that were used for this task include four categories representing Disease, Species, Drug/Proteins, and Drugs/Chemicals. The objective is to train and make a prediction on the labels, which are the proper nouns within the domain area. More formally, given an input sentence $S=\left\{x_{1}, x_{2}, \ldots, x_{z}\right\}$, where $x_{i}$ is the $i$-th word and $z$ represents the length of the sentence. The goal of BioNER is to classify each word in $S$ and assign it to a corresponding label $y \in Y$, where $Y$ is a predefined list of all possible label.

Fine-tuning is simple as compared to the pretraining, and the computational requirements are also not that significant. BioALBERT which takes less physical memory and improvised parameter sharing techniques. The BioNER fine-tuning is trained to learn the word embeddings using the sentence piece tokenization while the BioBERT model was based on the word piece embeddings. For each of the pre-trained models, we constructed a fine-tuning task by using the specific dataset.

The model setup uses the weights of the pre-trained model that are created previously. Adamw opitmzer and learning rate $(\mathrm{l}=0.00001)$ is used. Training batch size is 32 and evaluation batch size of 16 is used in our experiments. We restricted sentence length to 512 , lower-cased all words and saved model checked points at every 200 steps. Finally, we fine-tuned our pretrained models for 5,336 training steps and used 512 warm-up steps during fine-tuning process. All of the hyper-parameters used are same as default ALBERT unless stated otherwise. All the tested datasets contain a collection of words with a label "B" ("Beginning"), "I" ("Inside"), and "O" ("Outside") of an entity. For our experiments, we used these datasets as-is and passed to it our pre-trained models for the downstream task. The adamw optimiser was used with evaluation checkpoint so that the model will be evaluated at different time intervals using the holdout development dataset to identify the best model for final predictions. The predictions were performed on the test datasets, and the performance is compared with baseline models that were established previously by calculating the F1 Score, Precision and Recall. The summary of all parameters used in fine-tuning is given in Table 5 .
Table 5 Summary of parameters used in fine-tuning

\begin{tabular}{|c|c|}
\hline Summary of All parameters used: (Fine-Tuning) \\
\hline Name & BioNER \\
\hline Optimizer used & Adamw \\
\hline Training Batch Size Size & 32 \\
\hline Evaluation Batch Size Size & 16 \\
\hline Checkpoint Saved & 200 \\
\hline Maximum Sentence Length & 512 \\
\hline Learning Rate (Ir) & 0.00001 \\
\hline Training Steps & 5,336 \\
\hline Warm-up Steps & 320 \\
\hline
\end{tabular}

\section{Abbreviations}

BioNER: Biomedical Named Entity Recognition; NLP: Natural language processing; BiLSTM: Bi-directional long short-term memory network; ELMo: Embeddings from Language Model; CRF: Conditional random fields; BERT: Bi-directional encoder representations from transformer; ALBERT: A Lite Bidirectional Encoder Representations from Transformers; BioBERT: Biomedical Bi-directional encoder representations from transformer; BioALBERT: Biomedical A Lite Bidirectional Encoder Representations from Transformers; CDR: BioCreative $V$ chemical disease relation corpus; CID: Chemical-induced disease;

Ethics approval and consent to participate

Not applicable.

Consent for publication

Not applicable.

Availability of data and materials

The code and pre-trained models will be publicly available on GitHub at https://github.com/usmaann/BioALBERT (Under Construction)

Competing interests

The authors declare that they have no competing interests.

Funding

Not applicable.

\section{Authors' contributions}

UN designed the methodology. UN, VR and SR implemented the model, performed experiments and analyses. All authors participated in manuscript preparation. All authors read and approved the final manuscript.

\section{Acknowledgements}

Not applicable.

\section{Author details}

${ }^{1}$ School of Computer Science, University of Sydney, Australia. ${ }^{2}$ School of Information Technology, Deakin University, Australia. ${ }^{3}$ Telehealth and Technology Centre, Nepean hospital, Australia.

References

1. Meystre, S.M., Savova, G.K., Kipper-Schuler, K.C., Hurdle, J.F.: Extracting information from textual documents in the electronic health record: a review of recent research. Yearbook of medical informatics 17(01), 128-144 (2008)

2. Mårtensson, L., Hensing, G.: Health literacy-a heterogeneous phenomenon: a literature review. Scandinavian journal of caring sciences 26(1), 151-160 (2012)

3. Rosario, B., Hearst, M.: Classifying semantic relations in bioscience texts. In: Proceedings of the 42nd Annual Meeting of the Association for Computational Linguistics (ACL-04), Barcelona, Spain, pp. 430-437 (2004). doi:10.3115/1218955.1219010. https://www.aclweb.org/anthology/P04-1055

4. Lim, S., Lee, K., Kang, J.: Drug drug interaction extraction from the literature using a recursive neural network. PLOS ONE 13, 0190926 (2018). doi:10.1371/journal.pone.0190926

5. Cohen, A.M., Hersh, W.R.: A survey of current work in biomedical text mining. Briefings in bioinformatics 6(1), 57-71 (2005) 
6. Yadav, V., Bethard, S.: A Survey on Recent Advances in Named Entity Recognition from Deep Learning models (2019). 1910.11470

7. Peters, M.E., Neumann, M., lyyer, M., Gardner, M., Clark, C., Lee, K., Zettlemoyer, L.: Deep contextualized word representations. CoRR abs/1802.05365 (2018). 1802.05365

8. Devlin, J., Chang, M.-W., Lee, K., Toutanova, K.: BERT: Pre-training of deep bidirectional transformers for language understanding. In: Proceedings of the 2019 Conference of the North American Chapter of the Association for Computational Linguistics: Human Language Technologies, Volume 1 (Long and Short Papers), pp. 4171-4186. Association for Computational Linguistics, Minneapolis, Minnesota (2019). doi:10.18653/v1/N19-1423. https://www.aclweb.org/anthology/N19-1423

9. Lan, Z., Chen, M., Goodman, S., Gimpel, K., Sharma, P., Soricut, R.: ALBERT: A Lite BERT for Self-supervised Learning of Language Representations (2019). 1909.11942

10. Pyysalo, S., Ginter, F., Moen, H., Salakoski, T., Ananiadou, S.: Distributional semantics resources for biomedical text processing. (2013)

11. Jin, Q., Dhingra, B., Cohen, W.W., Lu, X.: Probing Biomedical Embeddings from Language Models (2019). 1904.02181

12. Lee, J., Yoon, W., Kim, S., Kim, D., Kim, S., So, C.H., Kang, J.: BioBERT: a pre-trained biomedical language representation model for biomedical text mining (2019). 1901.08746

13. Mikolov, T., Sutskever, I., Chen, K., Corrado, G.S., Dean, J.: Distributed representations of words and phrases and their compositionality. In: Advances in Neural Information Processing Systems, pp. 3111-9 (2013)

14. Chen, Q., Peng, Y., Lu, Z.: Biosentvec: creating sentence embeddings for biomedical texts. 2019 IEEE International Conference on Healthcare Informatics (ICHI), 1-5 (2018)

15. Zhu, H., Paschalidis, I.C., Tahmasebi, A.M.: Clinical concept extraction with contextual word embedding. NIPS Machine Learning for Health Workshop (2018)

16. Beltagy, I., Lo, K., Cohan, A.: SciBERT: A Pretrained Language Model for Scientific Text (2019). 1903.10676

17. Si, Y., Wang, J., Xu, H., Roberts, K.: Enhancing clinical concept extraction with contextual embeddings. Journal of the American Medical Informatics Association 26(11), 1297-1304 (2019). doi:10.1093/jamia/ocz096

18. Peng, Y., Yan, S., Lu, Z.: Transfer Learning in Biomedical Natural Language Processing: An Evaluation of BERT and ELMo on Ten Benchmarking Datasets (2019). 1906.05474

19. Howard, J., Ruder, S.: Universal language model fine-tuning for text classification. In: Proceedings of the 56th Annual Meeting of the Association for Computational Linguistics (Volume 1: Long Papers), pp. 328-339. Association for Computational Linguistics, Melbourne, Australia (2018). doi:10.18653/v1/P18-1031. https://www.aclweb.org/anthology/P18-1031

20. Yogatama, D., d'Autume, C.d.M., Connor, J., Kocisky, T., Chrzanowski, M., Kong, L., Lazaridou, A., Ling, W., Yu, L., Dyer, C., et al.: Learning and evaluating general linguistic intelligence. arXiv preprint arXiv:1901.11373 (2019)

21. Qiu, X., Sun, T., Xu, Y., Shao, Y., Dai, N., Huang, X.: Pre-trained models for natural language processing: A survey. arXiv preprint arXiv:2003.08271 (2020)

22. Liu, P., Qiu, X., Huang, X.: Recurrent neural network for text classification with multi-task learning. arXiv preprint arXiv:1605.05101 (2016)

23. Liu, X., He, P., Chen, W., Gao, J.: Multi-task deep neural networks for natural language understanding. arXiv preprint arXiv:1901.11504 (2019)

24. Xu, K., Yang, Z., Kang, P., Wang, Q., Liu, W.: Document-level attention-based bilstm-crf incorporating disease dictionary for disease named entity recognition. Computers in biology and medicine $\mathbf{1 0 8}$, 122-132 (2019)

25. Sachan, D.S., Xie, P., Sachan, M., Xing, E.P.: Effective Use of Bidirectional Language Modeling for Transfer Learning in Biomedical Named Entity Recognition (2017). 1711.07908

26. Lou, Y., Zhang, Y., Qian, T., Li, F., Xiong, S., Ji, D.: A transition-based joint model for disease named entity recognition and normalization. Bioinformatics (Oxford, England) 33 (2017). doi:10.1093/bioinformatics/bt×172

27. Luo, L., Yang, Z., Yang, P., Zhang, Y., Wang, L., Lin, H., Wang, J.: An attention-based bilstm-crf approach to document-level chemical named entity recognition. Bioinformatics 34, 1381-1388 (2018)

28. Yoon, W., So, C.H., Lee, J., Kang, J.: Collabonet: collaboration of deep neural networks for biomedical named entity recognition. BMC Bioinformatics 20(S10) (2019). doi:10.1186/s12859-019-2813-6

29. Giorgi, J.M., Bader, G.D.: Transfer learning for biomedical named entity recognition with neural networks. Bioinformatics 34(23), 4087-4094 (2018)

30. Doundefinedan, R.I., Leaman, R., Lu, Z.: Ncbi disease corpus. J. of Biomedical Informatics 47(C), 1-10 (2014)

31. Li, J., Sun, Y., Johnson, R.J., Sciaky, D., Wei, C.-H., Leaman, R., Davis, A.P., Mattingly, C.J., Wiegers, T.C., Lu, Z.: Biocreative v cdr task corpus: a resource for chemical disease relation extraction. Database : the journal of biological databases and curation 2016 (2016)

32. Krallinger, M., Rabal, O., Leitner, F., Vazquez, M., Salgado, D., lu, Z., Leaman, R., Lu, Y., Ji, D., Lowe, D., Sayle, R., Batista-Navarro, R., Rak, R., Huber, T., Rocktäschel, T., Matos, S., Campos, D., Tang, B., $\mathrm{Xu}, \mathrm{H}$., Valencia, A.: The chemdner corpus of chemicals and drugs and its annotation principles. Journal of Cheminformatics 7, 2 (2015). doi:10.1186/1758-2946-7-S1-S2

33. Ando, R.K.: Biocreative ii gene mention tagging system at ibm watson. (2007)

34. Kim, J.-D., Ohta, T., Tsuruoka, Y., Tateisi, Y., Collier, N.: Introduction to the bio-entity recognition task at jnlpba. In: Proceedings of the International Joint Workshop on Natural Language Processing in Biomedicine and Its Applications. JNLPBA '04, pp. 70-75. Association for Computational Linguistics, USA (2004)

35. Gerner, M., Nenadic, G., Bergman, C.M.: Linnaeus: a species name identification system for biomedical literature. BMC bioinformatics 11(1), 85 (2010)

36. Pafilis, E., Frankild, S.P., Fanini, L., Faulwetter, S., Pavloudi, C., Vasileiadou, A., Arvanitidis, C., Jensen, L.J.: The species and organisms resources for fast and accurate identification of taxonomic names in text. PLOS ONE 8(6), 1-6 (2013). doi:10.1371/journal.pone.0065390 


\section{Training Time BioALBERT v/s BioBERT}

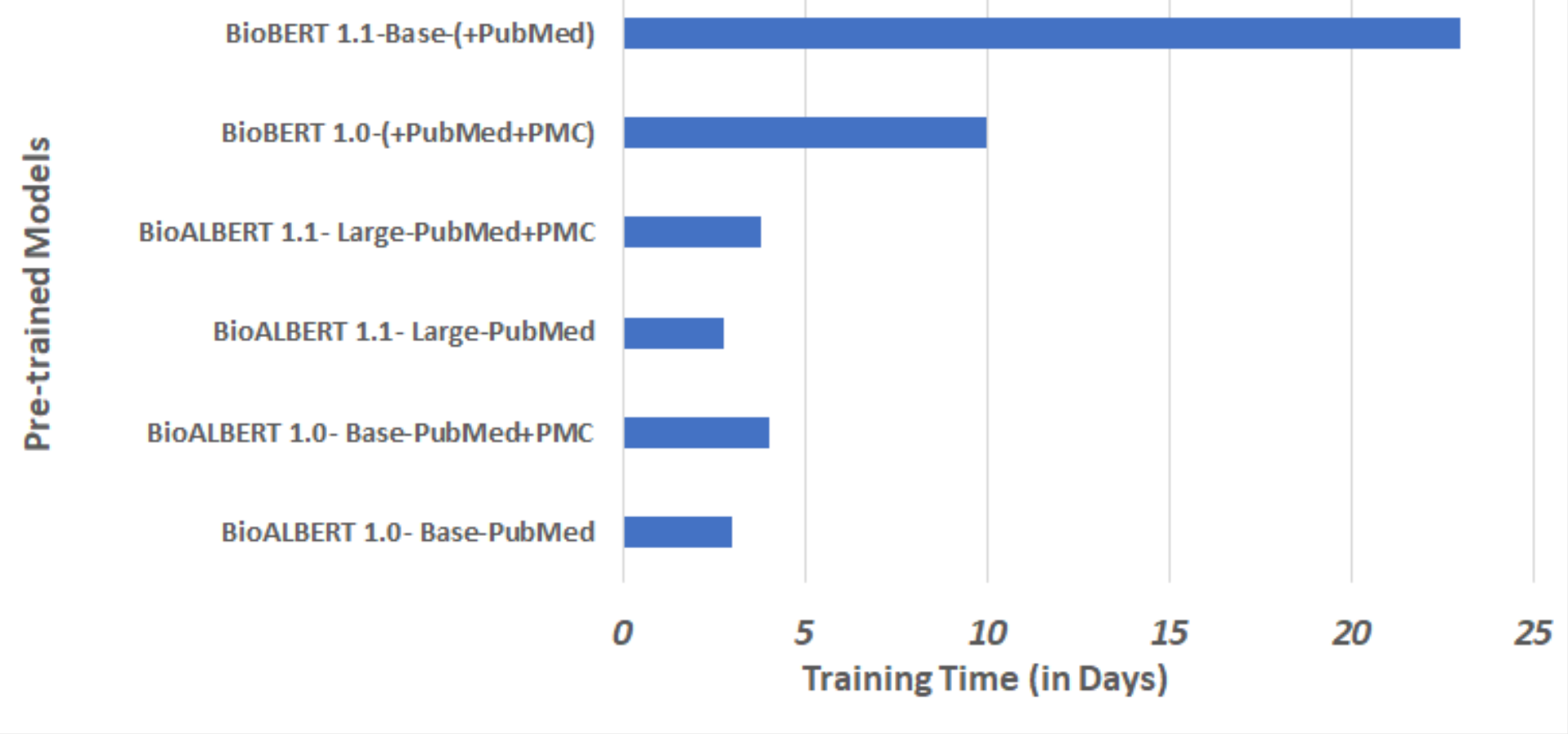

Figure 1

Comparison of run-time statistics of BioALBERT v/s BioBERT

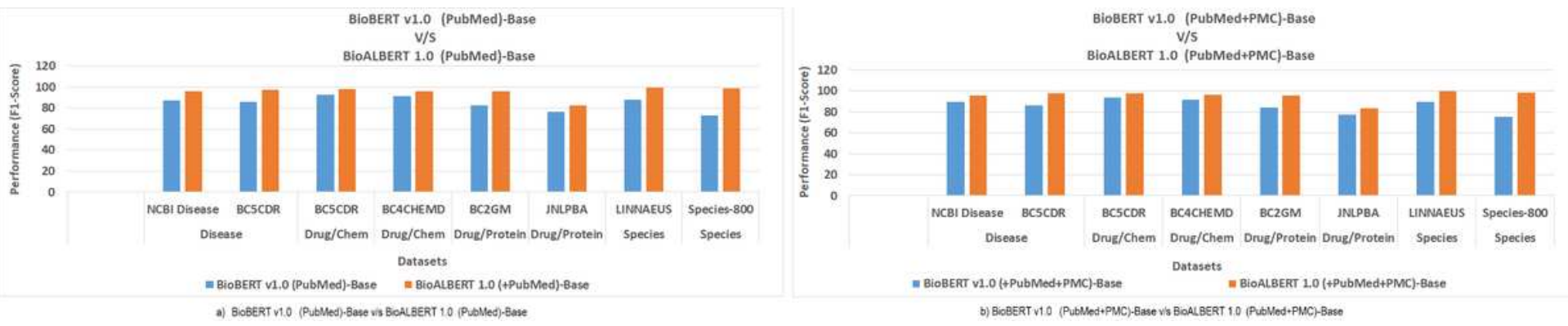

Figure 2

Comparison of a) BioBERT v1.0 (Pubmed)-Base v/s BioALBERT 1.0 (Pubmed)-Base ; b) BioBERT v1.0(Pubmed+PMC)-Base v/s BioALBERT 1.0(Pubmed+PMC)-Base 


\section{Analysis of BioALBERT models}

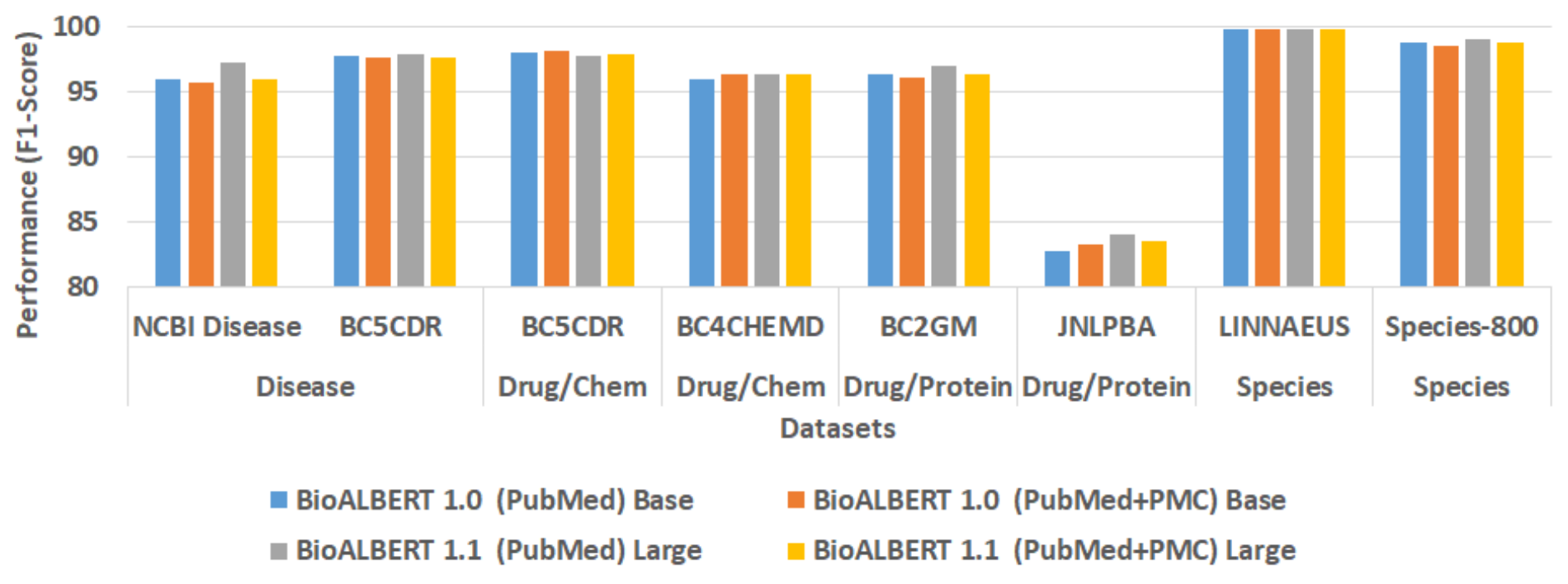

\section{Figure 3}

Comparison of different variants BioALBERT models

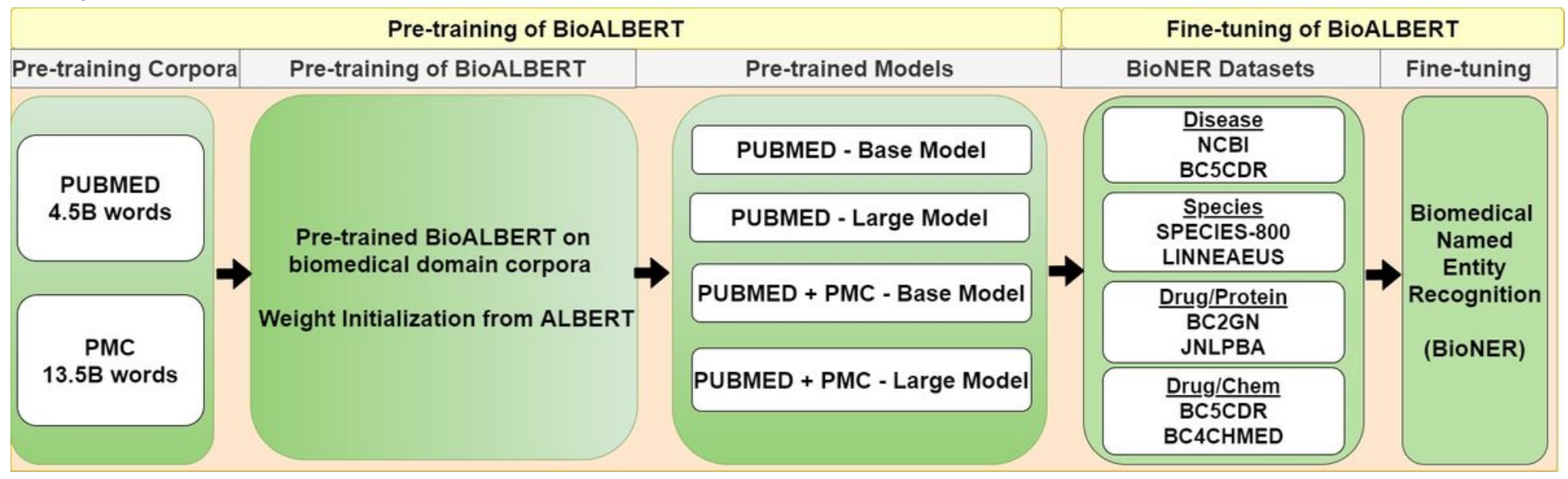

\section{Figure 4}

Overview of the pre-training and fine-tuning of BioALBERT on NER 